\title{
VALIDATION TESTS OF OPEN-SOURCE PROCEDURES FOR DIGITAL CAMERA CALIBRATION AND 3D IMAGE-BASED MODELLING
}

\author{
Isabella Toschi $^{\text {a }}$, Riccardo Rivola ${ }^{\text {a }}$, Eleonora Bertacchini ${ }^{\text {a }}$, Cristina Castagnetti ${ }^{\text {a }}$, Marco Dubbini ${ }^{\text {b }}$, Alessandro Capra ${ }^{\text {a }}$
}

\author{
a DIEF - Dept. of Engineering “Enzo Ferrari”, University of Modena and Reggio Emilia, \\ Via Vignolese 905, 41125 - Modena, Italy \\ www.ingmo.unimore.it \\ ${ }^{\mathrm{b}}$ DiSCi - Dept. of History Culture Civilization - Headquarters of Geography, University of Bologna, \\ Via Guerrazzi 20, 40125 - Bologna, Italy \\ www.storia-culture-civilta.unibo.it
}

KEY WORDS: Calibration, 3D Modelling, Accuracy, Close Range Photogrammetry, Open-source

\begin{abstract}
:
Among the many open-source software solutions recently developed for the extraction of point clouds from a set of un-oriented images, the photogrammetric tools Apero and MicMac (IGN, Institut Géographique National) aim to distinguish themselves by focusing on the accuracy and the metric content of the final result. This paper firstly aims at assessing the accuracy of the simplified and automated calibration procedure offered by the IGN tools. Results obtained with this procedure were compared with those achieved with a test-range calibration approach using a pre-surveyed laboratory test-field. Both direct and a-posteriori validation tests turned out successfully showing the stability and the metric accuracy of the process, even when low textured or reflective surfaces are present in the 3D scene. Afterwards, the possibility of achieving accurate 3D models from the subsequently extracted dense point clouds is also evaluated. Three different types of sculptural elements were chosen as test-objects and "ground-truth" data were acquired with triangulation laser scanners. 3D models derived from point clouds oriented with a simplified relative procedure show a suitable metric accuracy: all comparisons delivered a standard deviation of millimeter-level. The use of Ground Control Points in the orientation phase did not improve significantly the accuracy of the final 3D model, when a small figure-like corbel was used as test-object.
\end{abstract}

\section{INTRODUCTION}

The three-dimensional (3D) model of an object, even if it is a sculptural element rather than a more or less extensive portion of territory, is the result of a complex process, starting from data acquisition up to the management and sharing of a PCinteractive 3D virtual reconstruction of the scene. 3D modelling for Cultural Heritage (CH) documentation (Godin et al., 2002) has recently gained large diffusion and its applications range from digital conservation, accurate archiving and 3D catalogues up to cross-comparisons, computer-aided restoration and rapid prototyping. Various sensors and methods are available today to extract these models (Pavlidis et al., 2007). Among them, both Terrestrial Laser Scanners (TLS) and close-range photogrammetry can be efficiently used (Remondino, 2011). Comparisons and integration between range-based and imagebased techniques can be found in Beraldin (2004), Böhler and Marbs (2004), Guidi et al. (2009) and Remondino et al. (2005). Active sensors and range data (Blais, 2004; Vosselman and Maas, 2010) offer high level of automation and high geometric accuracy. These advantages have made TLS quite common in the last years in the $\mathrm{CH}$ field, despite their drawbacks, e.g. high cost, weight, considerable data collection and processing time and a lack of good texture.

On the contrary, passive sensors and image data (Remondino and El-Hakim, 2006) are nowadays receiving great attention from both the surveying community and non-experts, who benefit from the advantages of these systems, e.g. low cost, short data collection time, flexibility and direct extraction of photo-textured point clouds. In particular, the wide diffusion of high quality consumer-grade digital cameras, along with an increasing focus on photo-realistic 3D modelling of $\mathrm{CH}$, have recently forced both the photogrammetric and the computer vision communities to work together, in order to enhance the potential of the image-based approach. Since the beginning of this century, several algorithmic improvements have been achieved, such as:

- the introduction of tools like SIFT (Scale Invariant Feature Transform) (Lowe, 2004) or MSER (Maximally Stable Extremal Regions) (Matas et al., 2002) to extract dense features from overlapping images;

- the automatic orientation of large block of images using only tie points (Snavely et al., 2008);

- the multi-correlation and optimization techniques for dense matching of oriented images (Furukawa and Ponce, 2010).

The research has led to the development of many open-source software solutions for the extraction of point clouds from a set of un-oriented images, e.g. Bundler-PMVS (Snavely; Furukawa and Ponce), Photosynth (Microsoft Corporation), ARC3D (ARC3D), etc.

In this context, the French mapping agency (IGN - Institut Géographique National) delivered in 2007 several open-source photogrammetric tools with the aim of evidencing their accuracy and the quantitative content of their final results. Apero and MicMac (Apero, MicMac) are the two main tools being distributed: the first is a software for computing orientation of images (Pierrot-Deseilligny and Clery, 2011); the 
second is a tool for computing depth maps from oriented images with a multi-resolution image matching approach (PierrotDeseilligny and Paparoditis, 2006). Both tools require the adoption of specific photogrammetric rules in image acquisition as well as complex and parameterized procedures in image processing. However, the IGN also offers some simplified versions of these tools.

This paper firstly aims to assess the accuracy of the automated simplified calibration procedure offered by the IGN tools. Digital camera calibration (Remondino and Fraser, 2006) plays in fact a fundamental role in the photogrammetric pipeline for the extraction of precise and reliable metric information from images. Then, this research work evaluates the possibility of achieving accurate 3D models from the subsequently extracted dense point clouds. These validations were performed by comparisons with appropriate reference models. A Canon EOS 5D Mark II (5616 x 3744 px) mounting the zoom lens CANON EF $16-35 \mathrm{~mm}$ f2.8L USM was employed for the tests. Images were always recorded at fixed focal $(35 \mathrm{~mm})$ and focus settings.

\section{DIGITAL CAMERA CALIBRATION}

\subsection{Data Acquisition}

Validation tests of the calibration procedure were firstly performed using a pre-surveyed laboratory test-field (Figure 1). It is constituted by 38 targets, placed in the corner of a building and well distributed in three dimensions: their XYZ coordinates were measured by an automatic total station and statistically compensated with sub-millimetre accuracy.

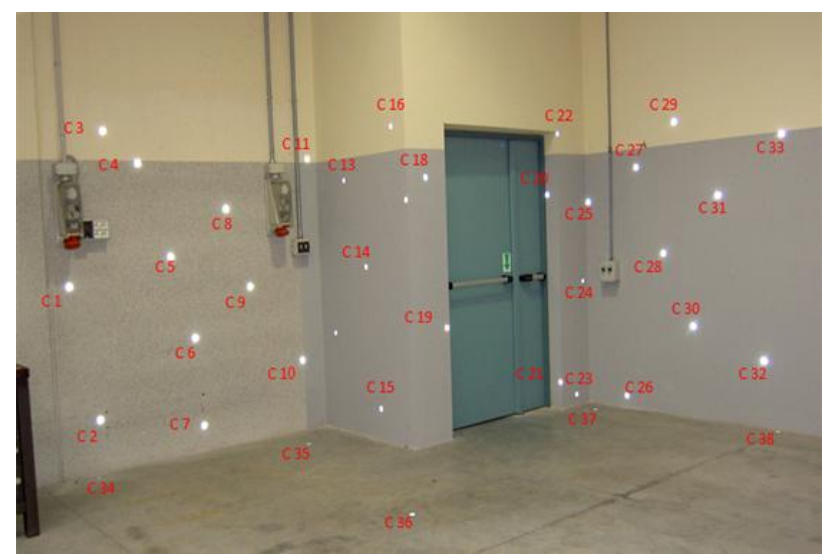

Figure 1. The calibration test-field

A total of 40 convergent images were acquired in conformance with the following photogrammetric rules: fixed zoom and focus setting (35 mm, infinity focus), multiple photo stations with varying camera-object distances, different roll angles (horizontal, vertical, oblique), great image point density and covering the entire image format with measured grid points. A photographic tripod was used and illumination conditions were artificially controlled during the acquisition phase. The images were then grouped into 20 different combinations, each containing the same number of shots (20), selected in order to follow the over mentioned basic photogrammetric rules.

Two additional tests were also performed without the use of a pre-surveyed control point grid, but employing different 3D scenes that may also include noise presence (e.g. reflective objects). The first test (Test 1) was carried out using a group of mechanical instruments and equipment as $3 \mathrm{D}$ scene, including some reflective materials. A building's low textured stairway (Figure 2) was then chosen as second test-site (Test 2).

A different number of shots were acquired in the two tests: 11 images for Test 1, 6 images for Test 2. In both cases, the acquisition phase was conducted following the above mentioned basic photogrammetric rules; natural conditions of illumination were used.

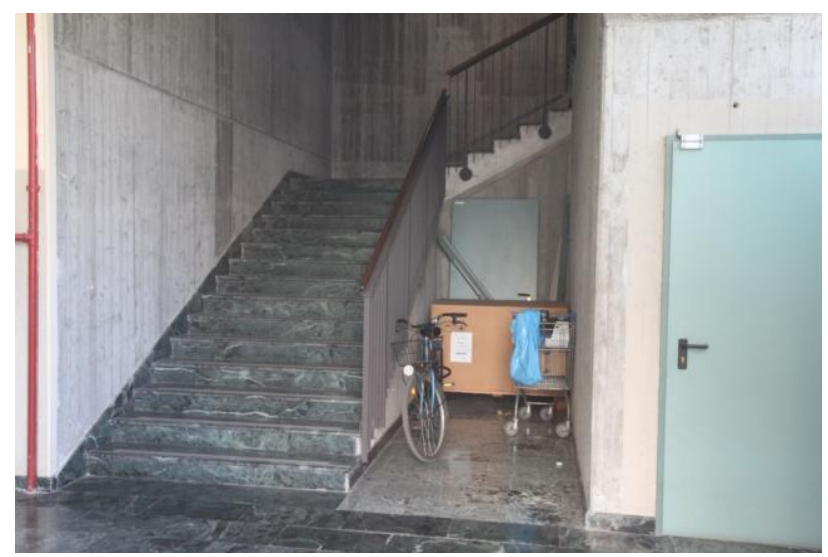

Figure 2. The building's stairway

\subsection{Data Processing}

The 20 test-field data-sets were initially processed using the scientific photogrammetric software MicroMap v. 2.0.0.143 (Geoin), released by Geoin. A test-range calibration (Fraser, 2011) with a general spatial resection approach was performed using the known XYZ object point coordinates as suitable control point test-field. Camera interior parameters were then computed and radial symmetrical distortion profiles were derived using a polynomial balanced formulation. This was carried out for each of the 20 image combinations; mean values and standard deviations were finally estimated.

The IGN photogrammetric tools were then employed to process the same 20 test-field data-sets. The first step of the process consisted of computing tie points between the images. This phase was conducted with the tool Tapioca, based on the Sift ${ }^{++}$ implementation of SIFT algorithm (Vedaldi 2010). All possible pairs of images were treated at their original resolution. Secondly, internal and external orientations were performed with the tool Tapas, a simplified version of the more complex tool Apero. Tapas computes relative orientations from the extracted tie points and performs self-calibration (Fraser, 2011) during the bundle adjustment procedure. This simplified tool does not allow the user to include external auxiliary information in the computation (e.g. Ground Control Point coordinates). This possibility is offered by the general tool Apero, that was later also used in tests for this research. Among the different proposed internal calibration models (Pierrot-Deseilligny and Clery, 2011), the general Fraser formulation was chosen (Fraser, 2011). For each data-set, a file containing the camera calibration parameters was then computed; radial symmetrical distortion profiles were derived using an odd-ordered polynomial series truncated at the seventh-order term. Mean values and standard deviations were finally estimated from the 20 combination results.

The simplified calibration procedure offered by the IGN tools was also used to process the images acquired in Test 1 and Test 2. Also in these cases, the same above mentioned procedural choices were adopted. 


\subsection{Results}

Two strategies were applied in order to compare the results achieved with the different software solutions.

Firstly, direct comparisons were performed considering both calibration results and derived statistical parameters. This required some mathematical operations aimed at making the results comparable and homogeneous: in particular, the Gaussian distortion profiles derived from Tapas calibration were properly balanced (Fraser, 2011). These direct validation assessments show a good match between the automated simplified calibration results (Tapas procedure) and those computed with the test-range calibration of MicroMap. This evidence occurs also for the achievements in Test 1 and Test 2. To verify these preliminary findings, a second validation strategy was carried out. A-posteriori validation tests were performed with the software MicroMap using two new images of the lab test-field, acquired in stereoscopic mode. This stereo pair was oriented with 7 well distributed targets of the test-field, chosen as Ground Control Points (GCPs); the inner orientation was performed using the most significant sets of calibration parameters computed with the two procedures. For the test-field data-sets, mean values were employed as well as results achieved in two specific image combinations: Combination 11 (MicroMap procedure) and Combination 8 (Tapas procedure). These were chosen among the others because their values of calibrated focal length, $f$, are closest to the mean value computed for the 20 combinations: in fact, at first analysis, $f$ appeared to be the most stable calibration parameter. In addition, MicroMap does not calculate the error associated with its calibration procedure, which could be eventually used as an alternative criterion.

Validation assessments were then carried out by comparing residuals computed on 8 well distributed targets of the testfield, chosen as Check Points (CPs), and their standard deviations. Residuals were calculated as the difference between the XYZ coordinates retrieved from the oriented stereoscopic model and those measured with total station.

The main results are listed in Table 3; in the raw labelled "Noncalibrated images" nominal values for the inner orientation (nominal focal length, no principal point offsets, no lens distortion) were used.

\begin{tabular}{|c|c|c|c|}
\cline { 2 - 4 } \multicolumn{1}{c|}{} & $\sigma_{\mathrm{x}}(\mathrm{m})$ & $\sigma_{\mathrm{y}}(\mathrm{m})$ & $\sigma_{\mathrm{z}}(\mathrm{m})$ \\
\hline $\begin{array}{c}\text { Non-calibrated } \\
\text { images }\end{array}$ & 0.033 & 0.130 & 0.112 \\
\hline $\begin{array}{c}\text { MicroMap: } \\
\text { mean values }\end{array}$ & 0.006 & 0.033 & 0.032 \\
\hline $\begin{array}{c}\text { MicMac: } \\
\text { mean values }\end{array}$ & 0.031 & 0.051 & 0.065 \\
\hline $\begin{array}{c}\text { MicroMap: } \\
\text { combination } 11\end{array}$ & 0.003 & 0.029 & 0.025 \\
\hline $\begin{array}{c}\text { MicMac: } \\
\text { combination } 8\end{array}$ & 0.019 & 0.041 & 0.041 \\
\hline Test 1 & 0.013 & 0.074 & 0.066 \\
\hline Test 2 & 0.004 & 0.069 & 0.070 \\
\hline
\end{tabular}

Table 3. Standard deviations, $\sigma(\mathrm{m})$, of the residuals computed on CPs

The use of computed calibration parameters greatly reduces the errors if compared to the test where nominal values have been employed. Both the MicroMap calibration and the Tapas procedure deliver standard deviations of few centimeters. Although the use of GCPs coordinates (MicroMap) appears to achieve the best results, the simplified calibration procedure (Tapas) is able to reach a comparable level of accuracy.

Moreover, even with "noisy" 3D objects, low textured surfaces and a lower number of images, the simplified and automated calibration procedure offered by the IGN tools shows a good stability and metric accuracy, as it was already pointed out from direct comparisons.

\section{3D IMAGE-BASED MODELLING}

\subsection{Data Acquisition}

After the calibration accuracy assessment, the dense matching from oriented images performed with the MicMac software was then evaluated. As test-objects, three different types of sculptural elements were chosen: a capital, a small figure-like corbel (Figure 4) and a medieval relief. They are all part of the sculptural heritage of the Cathedral of Modena (Italy). The choice of three different types of sculptures aims at evaluating the potential of the IGN tools in dealing with elements characterized by various extensions and depths. This paper focuses mainly on the results obtained for the corbel data-set.

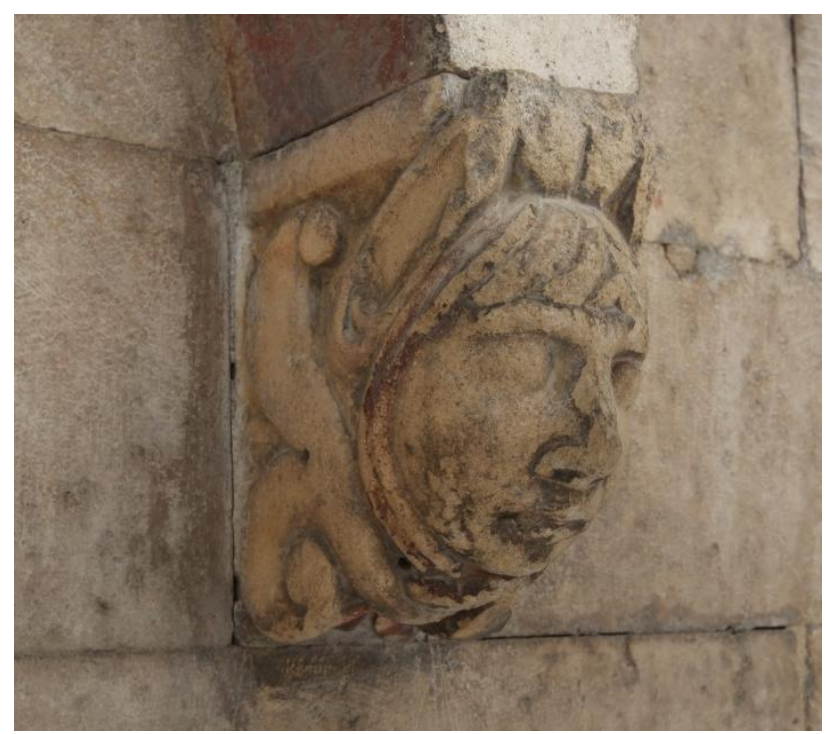

Figure 4 . The figure-like corbel (roughly 9x16x14 cm)

For the image collection of the corbel and the capital, a metallic scaffolding was employed to reach the required height (about 9 meters); the medieval relief was instead acquired directly from the ground. Focal $(35 \mathrm{~mm})$ and focus settings have been fixed during the acquisition of each element; a photographic tripod was used in the tests.

Each element was acquired following the general protocol recommended for terrestrial modelling (Pierrot-Deseilligny and Clery, 2011). For each point of view and, consequently, for each desired point cloud, a central "master" image was taken, together with other 3-4 closed associated images. A sufficient number of convergent shots were taken in order to assure the connection between each master image, with an overlap of around $80 \%$ between each pair of images. A reasonable base-todepth ratio was adopted to guarantee a good tie point detection without an excessive reduction of the final reconstruction 
accuracy. The number of acquired images depended consequently on the dimensions of the object and on external conditions, like the presence of obstacles. In particular, 12 images were sufficient to cover almost the entire surface of the corbel; the presence of the scaffold (above) and of the wall (sideways), however, prevented the shooting of the top and of some small side portions.

The "ground-truth" data were acquired with triangulation laser scanners: Faro CAM2 Platinum Scan Arm (nominal accuracy of $0.07 \mathrm{~mm}$ ) was used to acquire the 3D models of the capital and the corbel; the relief was instead surveyed by a Konica Minolta Range 7 (nominal accuracy of $0.04 \mathrm{~mm}$ ). For scans acquired on the scaffold, specific expedients were designed to reduce the external vibrations.

\subsection{Data processing}

The data processing phase was aimed at achieving detailed 3D surface models suitable for various applications, such as computer aided restoration and rapid prototyping. Both imagebased and range-based 3D modelling approaches were thereby performed.

The 3D modelling pipeline requested by the IGN tools consists mainly of three steps.

- The first phase is tie point extraction and was performed using the tool Tapioca. All possible pairs of images were processed at their original resolution.

- The second step is the bundle adjustment procedure (Triggs et al., 2000) for the computation of internal and external orientations. Initially, self-calibration (Fraser distortion formulation) and relative orientation were performed using the simplified tool Tapas. The Root Mean Square error (RMS) of the bundle adjustment was always lower than the pixel size; in particular, the RMS computed from the corbel data-set was 0.5 pixels. The corbel images were also oriented with the more complex tool Apero. It is constituted of four main modules (Pierrot-Deseilligny and Clery, 2011), i.e. computation of initial solution, bundle adjustment procedure, absolute geo-referencing and data import/export. If available, the user can include external information in the adjustment, like GCPs coordinates or GNSS (Global Navigation Satellite System) observations of the camera projection centre. In this test, absolute orientation was computed using GCP coordinates directly derived from the laser scanner model. For the calibration section, results computed via the previously performed Tapas calibration were declared as initial values; they were kept frozen at the beginning of the compensation and then re-evaluated. The final RMS of the bundle adjustment was 1.2 pixels.

- The third step of the procedure is, finally, the dense matching computation from oriented images. This phase was performed using the MicMac software with its multiscale, multi-resolution and pyramidal matching approach (Pierrot-Deseilligny and Paparoditis, 2006). The central "master" images collected during the acquisition phase were selected for the correlation procedure; the research area on each of them was defined through a masking process. The depth of field interval to be explored was set as well, starting from the orientation results. Computed depth maps were finally converted into 3D point clouds: this operation projects each pixel of the master image in the object space, using image orientation parameters and depth values. RGB attribute from master images is assigned to each $3 \mathrm{D}$ point.
Point clouds extracted with the image-based approach were then imported in Rapidform XOR3 (3D Systems), where the modelling process was performed using an automatic algorithm of mesh construction (3D Systems). Small holes were filled and defective flat surfaces were corrected. The final 3D models were finally exported in STereoLithography (STL) file format. Figure 5 shows an example of final 3D model (figure-like corbel).

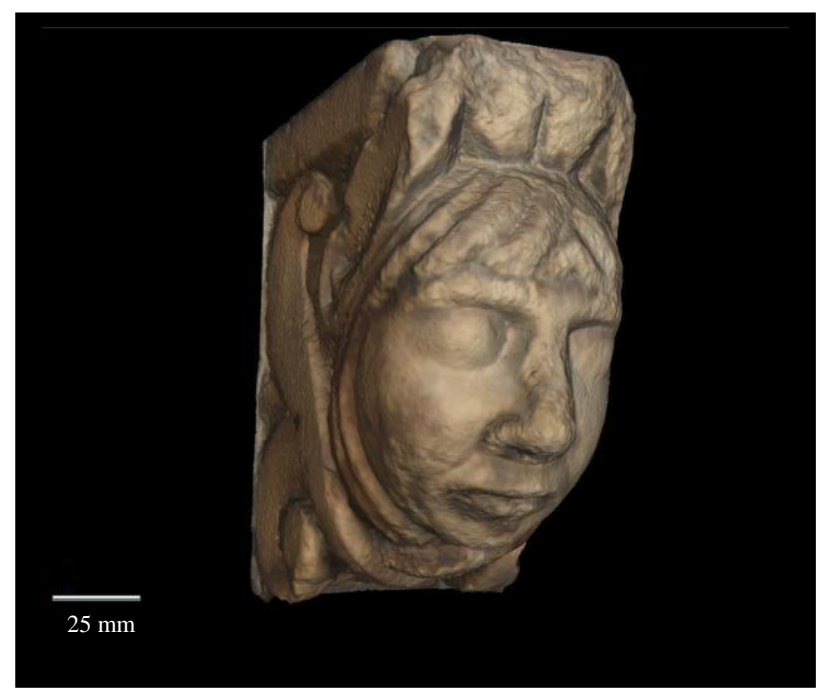

Figure 5. The 3D model of the figure-like corbel, obtained with the image-based approach and constituted by 3,920,000 triangular meshes

The range-based 3D modelling approach was performed in Rapidform XOR3, where scans acquired with triangulation laser scanners were processed. The final 3D models were considered to be suitable "ground-truth" data to check the accuracy of the image-based outputs.

\subsection{Results}

The metric accuracy of the final 3D models generated from point clouds extracted with the image-based approach was assessed through geometric comparisons with the above mentioned "ground-truth" data. These tests were performed within the open-source software CloudCompare (CloudCompare). Initially, data obtained from the relative orientation procedure (Tapas) were scaled and geo-referenced on corresponding laser scanner models. The registration phase was carried out in two steps: first, a roughly alignment was achieved through manually recognized pair points; secondly, an automatic refinement of the previous alignment was performed, applying the well known ICP (Iterative Closest Point) algorithm (Besl and McKay, 1992). The latter process showed always a millimeter-level accuracy: in particular, the RMS computed for the registration of the corbel 3D model was $1.09 \mathrm{~mm}$. Finally, geometric distances between the vertices of the image-based models and the range-based ones were computed with associated statistics.

All comparisons delivered a standard deviation of the differences between the data-sets of millimeter-level. In particular, some statistical parameters obtained for the corbel data-set are listed in Table 6; both 3D models derived from point clouds oriented with the relative simplified procedure (Tapas-derived 3D model) and with the absolute one (Aperoderived 3D model) were metrically evaluated. 


\begin{tabular}{|c|c|c|}
\cline { 2 - 3 } \multicolumn{1}{c|}{} & $\begin{array}{c}\text { Tapas-derived } \\
\text { 3D model }\end{array}$ & $\begin{array}{c}\text { Apero-derived } \\
\text { 3D model }\end{array}$ \\
\hline $\begin{array}{c}\text { Mean distance } \\
(\mathrm{mm})\end{array}$ & -0.04 & -0.06 \\
\hline $\begin{array}{c}\text { Standard } \\
\text { deviation }(\mathrm{mm})\end{array}$ & 0.51 & 0.46 \\
\hline $\begin{array}{c}\text { Positive } \\
\text { maximum }(\mathrm{mm})\end{array}$ & 4.90 & 4.91 \\
\hline $\begin{array}{c}\text { Negative } \\
\text { maximum }(\mathrm{mm})\end{array}$ & -3.44 & -3.48 \\
\hline
\end{tabular}

Table 6. Comparison between the corbel image-based 3D models and the "ground-truth" data: statistical results

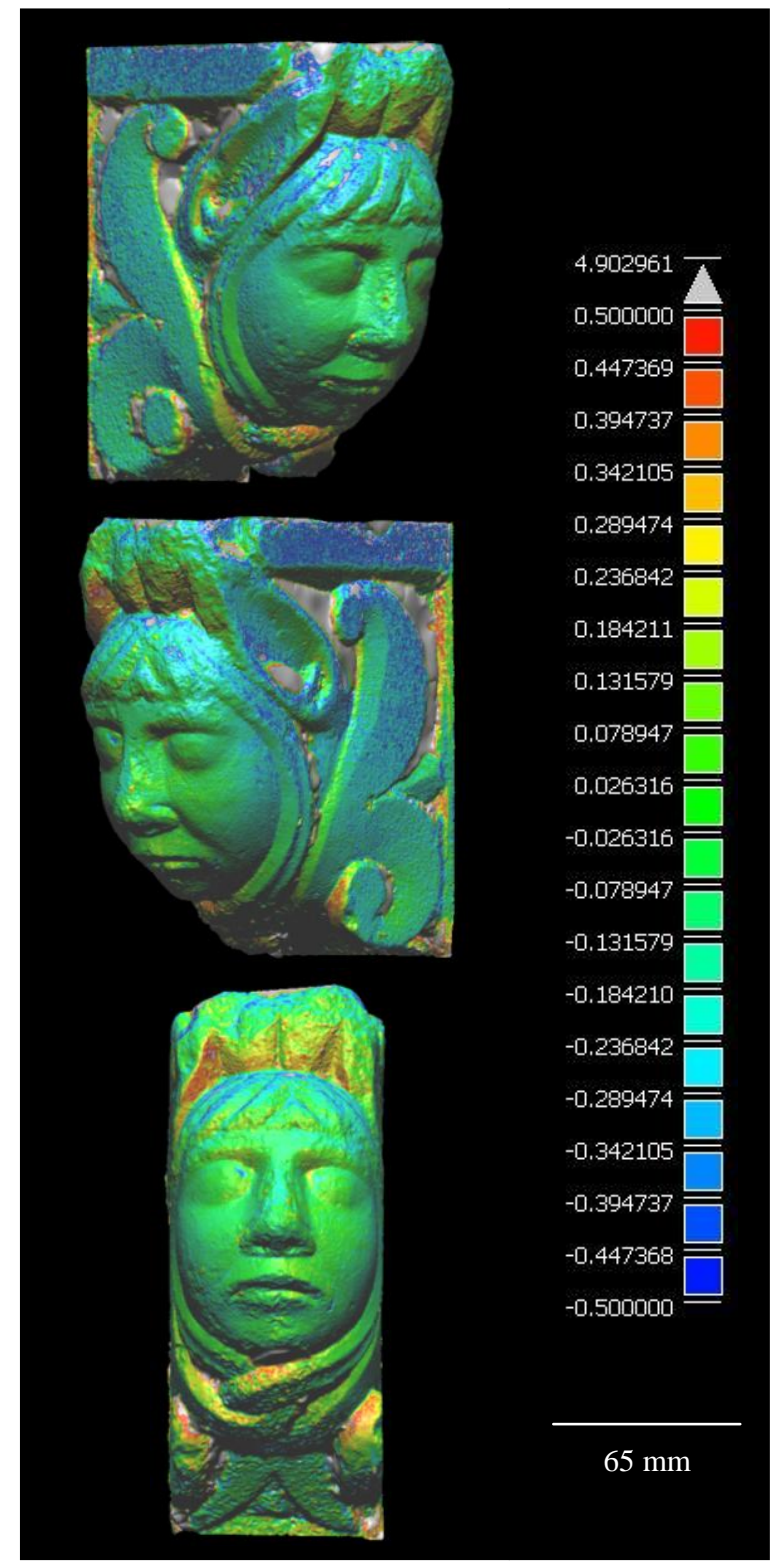

Figure 7. Comparison between the corbel 3D model (Tapasderived) and the "ground-truth" data: deviation map (mm)

Figure 7 shows three views of the deviation map obtained from the comparison between the Tapas-derived 3D model and the "ground-truth" data, for the corbel test-object. The colour scale ranges from $-0.5 \mathrm{~mm}$ (blue) to $0.5 \mathrm{~mm}$ (red): this distance interval is chosen in accordance with the standard deviation delivered by the comparison. All points of distance values falling outside this range are coloured in grey.

Both 3D models extracted with the IGN tools from the corbel data-set show the same level of metric accuracy, if compared with the same reference model: this evidence points out that for simple and small objects even the simplified procedure of calibration and relative orientation (Tapas) works successfully and is sufficient to achieve a suitable accuracy level.

For both models (Tapas-derived and Apero-derived), the portions showing greatest deviations from the "ground-truth" data correspond to those parts that were not directly acquired by the digital camera due to the presence of obstacles (top and some side portions). Those small areas were instead acquired with the triangulation laser scanner, making the reference model complete everywhere.

\section{CONCLUSIONS AND FUTURE WORK}

The validation tests described in this paper show the metric potentiality of the IGN tools for accurate and detailed 3D reconstruction with an image-based approach.

The accuracy of the simplified and automated calibration procedure (Tapas) was evaluated through comparisons with results achieved with a test-range calibration approach using a pre-surveyed laboratory test-field. The Fraser's distortion formulation was chosen among the many calibration models offered by the IGN tools. Different 3D scenes were used as testside, simulating the presence of low-textured surfaces (laboratory test-field data-sets and Test 2) and reflective materials (Test 1). Both direct and a-posteriori validations turned out successfully showing the stability and metric accuracy of the process. Anyway, although the simplified calibration procedure proved to be satisfactory, further tests will be performed in the future to verify if the combined use of Apero and external GCPs may enhance the accuracy of the calibration final results. Alternative internal calibration models will be tested as well.

Later on, the possibility of achieving an accurate 3D model from the subsequently extracted dense point clouds was also evaluated. Three different types of sculptural elements were chosen as test-objects and "ground-truth" data were acquired with triangulation laser scanners. All 3D models derived from point clouds oriented with the simplified relative procedure (Tapas) show a suitable metric accuracy: in particular, the comparison performed with the corbel data-set delivered a standard deviation of the differences between the compared models of $0.51 \mathrm{~mm}$. The use of Apero and laser scanner GCPs in the orientation phase, did not improve significantly the accuracy of the final 3D model. Future works will be addressed to evaluate if the simplified procedure of calibration and relative orientation (Tapas) is suitable even for more complex and extended objects and which circumstances require preferably the use of Apero with external information (like GCP coordinates or GNSS observations). The qualitative analysis of deviation maps extracted from comparisons show that occluded area cause the most remarkable errors. In the future, more robust statistical analysis on the population of distance values provided by the comparisons will be performed. Finally, detailed studies will be carried out in order to investigate which error sources affect each step of the procedural pipeline, from tie point detection up to the final 3D model extraction. 


\section{REFERENCES}

3D Systems. Rapidform XOR - User Guide. www.rapidform.com (1 Jul., 2013).

Apero, MicMac. http://www.micmac.ign.fr/ (1 Jul., 2013).

ARC3D. Automatic Reconstruction Cloud. http://www.arc3d.be/ (1 Jul., 2013).

Beraldin, J-A., 2004. Integration of laser scanning and close range photogrammetry - the last decade and beyond. In: Proceedings of the XXth International Society for Photogrammetry and Remote Sensing Congress, Istanbul, Turkey, pp. 972-983.

Besl, P.J., McKay, N.D., 1992. A method for registration of 3D shape. IEEE Transactions on Pattern Analysis and machine Intelligence, 14 , pp. 239-254.

Blais, F., 2004. Review of 20 years of range sensors development. Journal of Electronic Imaging, 13(1), pp. 231243.

Böhler, W., Marbs, A., 2004. 3D scanning and photogrammetry for heritage recording: a comparison. In: Proceedings of the XIIth International Conference on Geoinformatics, Gävle, Sweden, pp. 291-198.

CloudCompare. http://www.danielgm.net/cc/ (1 Jul., 2013).

Fraser, C.S., 2001. Photogrammetric Camera Component Calibration: A Review of Analytical Techniques. In: Calibration and Orientation of cameras in Computer Vision, Gruen and Huang (Eds.), Springer Series in Information Sciences 34, pp. 95-121.

Furukawa, Y., Ponce, J. PMVS: Patch-based Multi-viewStereo Software. http://www.di.ens.fr/pmvs/ (1 Jul., 2013).

Furukawa, Y., Ponce, J., 2010. Accurate, dense and robust multi-view stereopsis. IEEE Trans. On Pattern Analysis and Machine Intelligence, 32(8), pp. 1362-1376.

Geoin. MicroMap. http://www.geoin.it/ (1 Jul., 2013).

Godin, G., Beraldin, J.-A., Taylor, J., Cournoyer, L., Rioux, M., El-Hakim, S., Baribeau, R., Blais, F., Boulanger, P., Domey, J., Picard, M., 2002. Active Optical 3D Imaging for Heritage Applications. Computer Graphics and Applications, 22(5), pp. 24-35.

Guidi, G., Remondino, F., Russo, M., Menna, F., Rizzi, A., Ercoli, S., 2009. A multi-resolution methodology for the 3D modelling of large and complex archeological areas. International Journal of Architectural Computing, 7(1), pp.3955.

Lowe, D.G., 2004. Distinctive image features from scaleinvariant keypoints. International Journal of Computer Vision, 60(2), pp. 91-110.

Matas, J., Chum, O., Urban, M., Pajdla, T., 2002. Robust wide baseline stereo from maximally stable extremal regions. In: Proceedings of the British Machine Vision conference, Cardiff, UK, pp. 384-396.
Microsoft Corporation. Microsoft Photosynth. http://photosynth.net (1 Jul., 2013).

Pavlidis, G., Koutsoudis, A., Arnaoutoglou, F., Tsioukas, V., Chamzas, C., 2007. Methods for 3D digitization of Cultural Heritage. Journal of Cultural Heritage, 8, pp. 93-98.

Pierrot-Deseilligny, M., Clery, I., 2011. APERO, an opensource bundle adjustment software for automatic calibration and orientation of set of images. In: The International Archives of the Photogrammetry, remote Sensing and Spatial Information Sciences, ISPRS Int. Workshop 3D-ARCH 2011, Trento, Italy.

Pierrot-Deseilligny, M., Paparoditis, N., 2006. A multiresolution and optimization-based image matching approach: an application to surface reconstruction from SPOT5HRS stereo imagery. In: The International Archives of the Photogrammetry, remote Sensing and Spatial Information Sciences, ISPRS Workshop on Topographic Mapping from Space, Ankara, Turkey, pp. 73-77.

Remondino, F., 2011. Heritage recording and 3D modelling with photogrammetry and 3D scanning. Remote Sensing, 3(6), pp. 1104-1138.

Remondino, F., El-Hakim, S., 2006. Image-based 3D modelling: a review. The photogrammetric Record, 21(115), pp. 269-291.

Remondino, F., Fraser, C., 2006. Digital camera calibration methods: considerations and comparisons. In: The International Archives of the Photogrammetry, remote Sensing and Spatial Information Sciences, Dresden, Germany, pp. 266-272.

Remondino, F., Guarnieri, A., Vettore, A., 2005. 3D modelling of close range objects: photogrammetry or laser scanning? In: Proceedings of SPIE-IS\&T Electronic Imaging: Videometrics VIII, San Jose, California, Vol. 5665, pp. 216-225.

Snavely, N. Bundler: Structure from motion for unordered image collections. http://www.cs.cornell.edu/ snavely/bundler/ (1 Jul., 2013).

Snavely, N., Seitz, S., Szeliski, R., 2008. Modelling the world from internet photo collections. International Journal of Computer Vision, 80(2), pp. 189-210.

Triggs, B., McLauchlan, P., Hartley, R., Fitzgibbon, A., 2000. Bundle adjustment - a modern synthesis. In Vision Algorithms: Theory and Practice, Lecture Notes in Computer Science 1883, Springer-Verlag, Berlin, Germany, pp. 298-.372.

Vedaldi 2010. http://www.vlfeat.org/ vedaldi/code/siftpp.html (1 Jul., 2013).

Vosselman, G., Maas, H-G., 2010. Airborne and terrestrial laser scanning. CRC, Boca Raton, pp. 318. 